\title{
Structure and function of the Arctic and Antarctic marine microbiota as revealed by metagenomics
}

\author{
Shunan $\mathrm{Cao}^{1 \dagger}$, Weipeng Zhang ${ }^{2 \dagger}$, Wei Ding ${ }^{3}$, Meng Wang ${ }^{2}$, Shen Fan², Bo Yang ${ }^{2}$, Andrew Mcminn ${ }^{2}$, Min Wang ${ }^{2}$, \\ Bin-bin Xie ${ }^{3}$, Qi-Long Qin ${ }^{3}$ Xiu-Lan Chen ${ }^{3}$, Jianfeng $\mathrm{He}^{1^{*}}$ and Yu-Zhong Zhang ${ }^{2^{*}}$
}

\begin{abstract}
Background: The Arctic and Antarctic are the two most geographically distant bioregions on earth. Recent sampling efforts and following metagenomics have shed light on the global ocean microbial diversity and function, yet the microbiota of polar regions has not been included in such global analyses.

Results: Here a metagenomic study of seawater samples $(n=60)$ collected from different depths at 28 locations in the Arctic and Antarctic zones was performed, together with metagenomes from the Tara Oceans. More than 7500 (19\%) polar seawater-derived operational taxonomic units could not be identified in the Tara Oceans datasets, and more than 3,900,000 protein-coding gene orthologs had no hits in the Ocean Microbial Reference Gene Catalog. Analysis of 214 metagenome assembled genomes (MAGs) recovered from the polar seawater microbiomes, revealed strains that are prevalent in the polar regions while nearly undetectable in temperate seawater. Metabolic pathway reconstruction for these microbes suggested versatility for saccharide and lipids biosynthesis, nitrate and sulfate reduction, and $\mathrm{CO}_{2}$ fixation. Comparison between the Arctic and Antarctic microbiomes revealed that antibiotic resistance genes were enriched in the Arctic while functions like DNA recombination were enriched in the Antarctic.

Conclusions: Our data highlight the occurrence of dominant and locally enriched microbes in the Arctic and Antarctic seawater with unique functional traits for environmental adaption, and provide a foundation for analyzing the global ocean microbiome in a more complete perspective.
\end{abstract}

Keywords: Global ocean microbiome, Arctic and Antarctic zones, Environmental adaptation

\section{Introduction}

Recent advances in molecular ecological techniques, such as metagenomics, have enabled a more efficient pathway to address the taxonomic and functional composition of marine microbiota, including the free-living bacterioplankton

\footnotetext{
* Correspondence: hejianfeng@pric.org.cn; zhangyuzhong6830@ouc.edu.cn ${ }^{\dagger}$ Weipeng Zhang and Shunan Cao contributed equally to this work.

${ }^{1}$ The Key Laboratory for Polar Science MNR, Polar Research Institute of China, Shanghai 200136, China

${ }^{2}$ College of Marine Life Science, Frontiers Science Center for Deep Ocean Multispheres and Earth System, Ocean University of China, Qingdao 266003, China

Full list of author information is available at the end of the article
}

[1-3], the sediment-dwelling microbes [4-6], the surfaceattached biofilms [7-9], and the animal-associated symbionts [10-12]. Pursuing the goal characterizing the global seawater microbiota, the Tara Oceans expedition collected seawater samples from 68 locations, representing all main oceanic regions, and performed metagenomics to study the structure and function of the global ocean microbiota [1]. As a result, global ocean microbial diversity has been systematically investigated, and found to consist of more than 35,000 microbial "species" [1]. It was noted that depth is the major factor structuring the microbial community, explaining $73 \%$ of the variance in composition [1]. More

(c) The Author(s). 2020, corrected publication May 2020. Open Access This article is licensed under a Creative Commons Attribution 4.0 International License, which permits use, sharing, adaptation, distribution and reproduction in any medium or format, as long as you give appropriate credit to the original author(s) and the source, provide a link to the Creative Commons licence, and indicate if changes were made. The images or other third party material in this article are included in the article's Creative Commons licence, unless indicated otherwise in a credit line to the material. If material is not included in the article's Creative Commons licence and your intended use is not permitted by statutory regulation or exceeds the permitted use, you will need to obtain permission directly from the copyright holder. To view a copy of this licence, visit http://creativecommons.org/licenses/by/4.0/. The Creative Commons Public Domain Dedication waiver (http:// creativecommons.org/publicdomain/zero/1.0/) applies to the data made available in this article, unless otherwise stated in a credit line to the data. 
importantly by constructing an ocean microbial reference gene catalog (OM-RGC), the Tara Oceans project identified ubiquitous genes that are enriched for functions including coenzyme, lipid, nucleotide, amino acids, and secondary metabolites transport [1]. However, one shortcoming of this holistic global ocean study is that no Arctic samples and only five Antarctic samples were included.

The Arctic and Antarctic are two of the most geographically distant bioregions on Earth. The two regions have similar physical-chemical characteristics; characterized by low temperature, low availablility of carbon sources, and extreme seasonality in light conditions [13, 14]. Attention has been paid to the changes caused by climate change in both of the polar regions, such as the record of water freshening $[15,16]$ and greenhouse gas emission $[17,18]$. However, there are also differences; surface temperatures over much of the Arctic are continually increasing while at the moment major increases in Antarctic temperatures are limited to the Antarctic Peninsula area [19-21]; there is even record of increasing sea-ice coverage in the Antarctic based on satellite-derived observations from 1979 to 2015 [22].

Over the past decades there have been a number of attempts to understand the distribution of microbioal taxa in the polar oceans. Galand et al. [23] focused on rare taxa in Arctic seawater and highlighted the role of ecological processes such as selection and extinction on their biogeography. Ghiglione et al. [24] studied the taxonomic structures of microbiota in Arctic and Antarctic water and highlighted the difference between polar and non-polar seawater microbiota, as $78 \%$ of operational taxonomic units (OTUs) were unique to the Southern Ocean and $70 \%$ were unique to the Arctic Ocean; consistently, the finding by Kleinteich et al. highlighted the pole-to-pole connections of the seawater microbiota [25]. It was also found that deep ocean communities differed less between polar and nonpolar waters in comparison with the surface ocean communities [24]. By sampling marine epipelagic bacterial communities from the Arctic, Atlantic, Pacific, and Southern oceans, the study by Sul et al. [26] revealed latitudinal gradient in bacterial diversity.

Despite the previous efforts, a systematic understanding of the structure of polar microbiota and its place in a global context is lacking; more importantly, as most of the studies were based on 16S rRNA gene amplicon sequencing, functional characteristics linking microbial adaptation with the polar environment remains elusive. Thus, the aim of the present study is to address the following questions: (i) how does endemism in polar regions compare with elsewhere in the world; (ii) what are the specific functions of the polar microbiota, and how do these functions contribute to environmental adaptation. During the cruises to the Arctic and Antarctic, 60 samples were collected from 28 sites across multiple depths. The samples underwent metagenomic sequencing and were compared with data from the Tara Oceans project.

\section{Results and Discussion}

Structure and diversity of the polar microbiota

Sampling locations are shown in Fig. 1. A total of 39 Arctic seawater and 21 Antarctic seawater samples were collected for this project. Based on the sample depth, the samples were further divided into four groups: Arctic-Surface (0$100 \mathrm{~m})$, Arctic-Deep (200-4000 m), Antarctic-Surface (0$100 \mathrm{~m})$, and Antarctic-Deep (200-4000 m). Correspondingly, 60 metagenomes were obtained after Illumina sequencing. 16S rRNA gene sequences were extracted from the metagenomes for analysis and are subsequently referred to as $16 \mathrm{~S}$ miTags. Classification of OTUs at $97 \%$ similarity resulted in a total of 24,504 OTUs. Classification of OTUs at the phylum level (class level for Proteobacteria) (Fig. 2) revealed 90 taxa, with a dominance by Alphaproteobacteria and Gammaproteobacteria in most of the metagenomes, followed by Bacteroidetes. In addition, Deltaproteobacteria, Actinobacteria, Cyanobacteria, Crenacrchaeota, OD1 (Parcubacteria), and SAR406 (Marinimicrobia) showed high relative abundances in certain metagenomes. When the OTUs were classified at genus level (Additional file 1: Figure S1), 2,121 genera were obtained, dominated by an unnamed Pelagibacteraceae group. A total of $12.63 \pm 0.06 \%$ of the reads could not be classified to genus level.

To examine the similarity between the Arctic, Antarctic and the non-polar seawater microbial communities, 55 surface seawater $(5 \mathrm{~m})$ and 19 deep seawater metagenomes (100-1000 m) were downloaded from the Tara Oceans project [1] (http://ocean-microbiome.embl.de/companion. html) for comparison. Alpha-diversity analysis (Fig. 3a), based on rarefied $16 \mathrm{~S}$ miTags $(10,000$ miTags per metagenome), revealed an overall higher Chaol diversity, Shannon diversity, and Simpson diversity for the polar microbial communities; the alpha-diversities in the deep seawater samples tended to be higher than those in the surface seawater. Principal coordinates analysis (PCoA) (Fig. 3b) using rarefied $16 \mathrm{~S}$ miTag data revealed clear separation of the polar from the non-polar seawater microbiota, especially for the surface microbiota, with PCo1 explaining 25.14\% of the variability. Interestingly, the Arctic-Surface and the Antarctic-Surface seawater were clustered together, as were the Arctic-Deep and Antarctic-Deep seawater (Fig. 3b).

As found in previous studies based on 16S rRNA gene amplicon sequencing [23-26], the microbiota of Arctic and Antarctic seawater are more similar to each other than to seawater from other regions, and the dissimilarity between polar and temperate communities is more marked at the surface than in deeper water. Consistent with these findings, metagenomic analyses performed in the present study revealed a high similarity between microbiota in the two polar regions, which is likely to be attributed to 


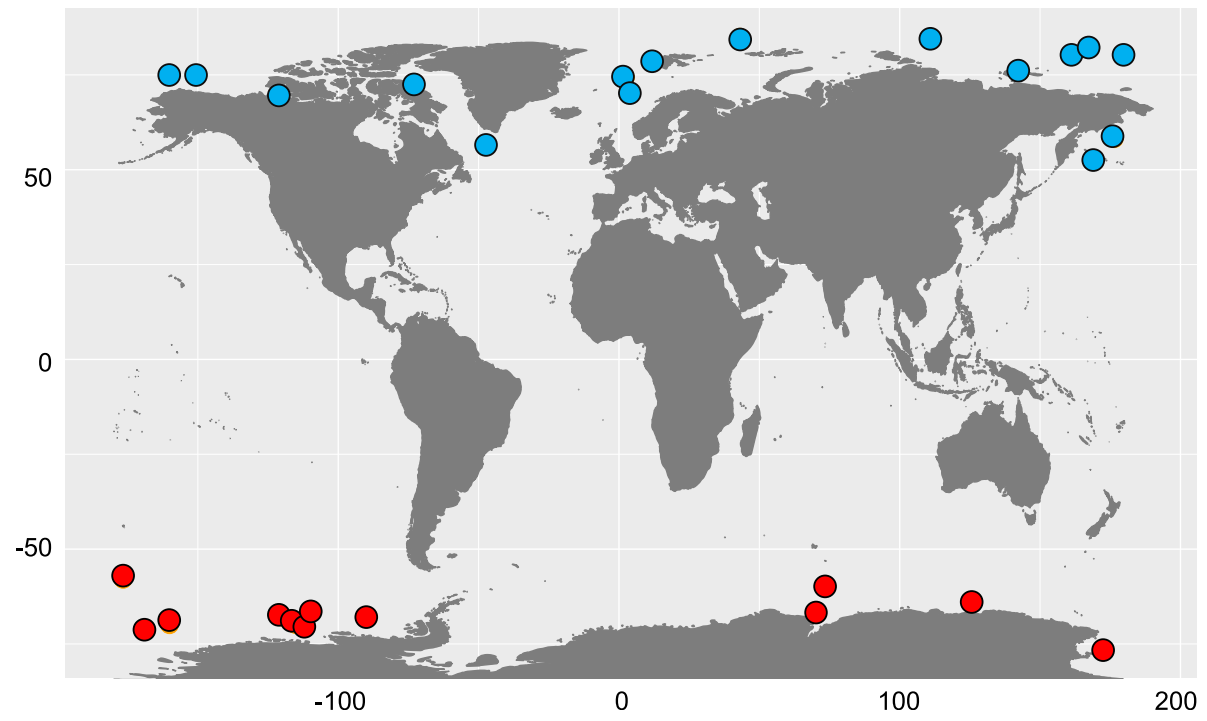

Fig. 1 Geographic distribution of the 28 sampling locations of the Arctic (blue) and Antarctic (red) seawater

environmental filtering (effect of environmental variables on community composition) and microbial dispersal, as the environmental conditions in the Arctic and the Antarctic are more similar to each than to the temperate regions. Higher diversity of microbes in deep polar seawater than in surface seawater was observed, implying that the deep waters are more of a reservoir of microbial species. Moreover, a higher similarity between the deep waters of temperate and polar area waters than between the surface waters of polar and temperate areas were found, which can be explained by the fact that surface communities have a high phylogenetic turnover rate [27] and minimal

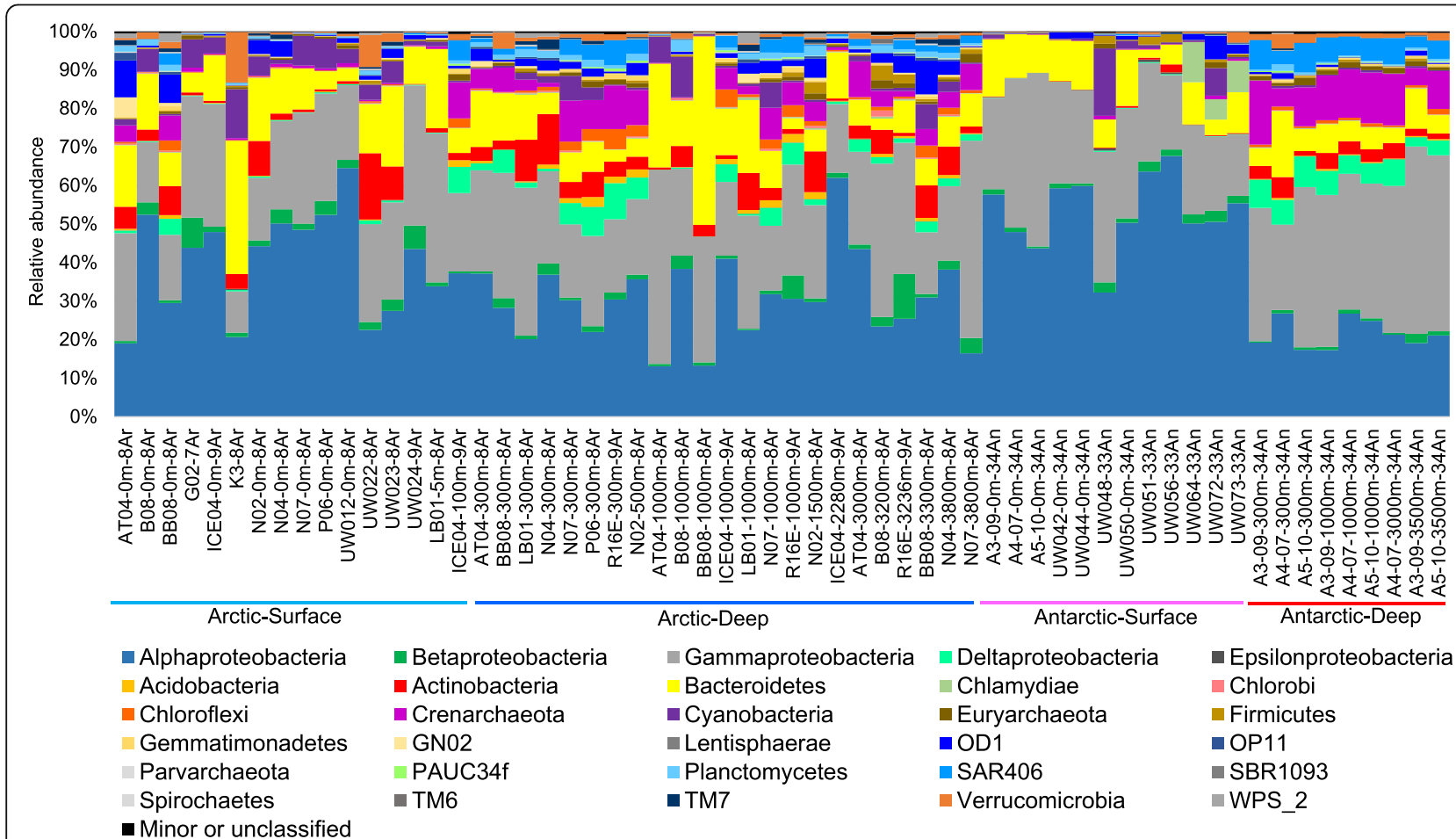

Fig. 2 Phylum-level (class level for Proteobacteria) taxonomic structure of the 60 polar seawater metagenomes. Relative abundance of the phyla was calculated based on 165 miTag numbers. The top 30 phyla in terms of maximum relative abundance among the metagenomes are shown with all other phyla and the unclassified miTags are grouped together as "Minor or unclassified." The four groups: Arctic-Surface (0-100 m), Arctic-Deep (200-4000 m), Antarctic-Surface (0-100 m), and Antarctic-Deep (200-4000 m) 

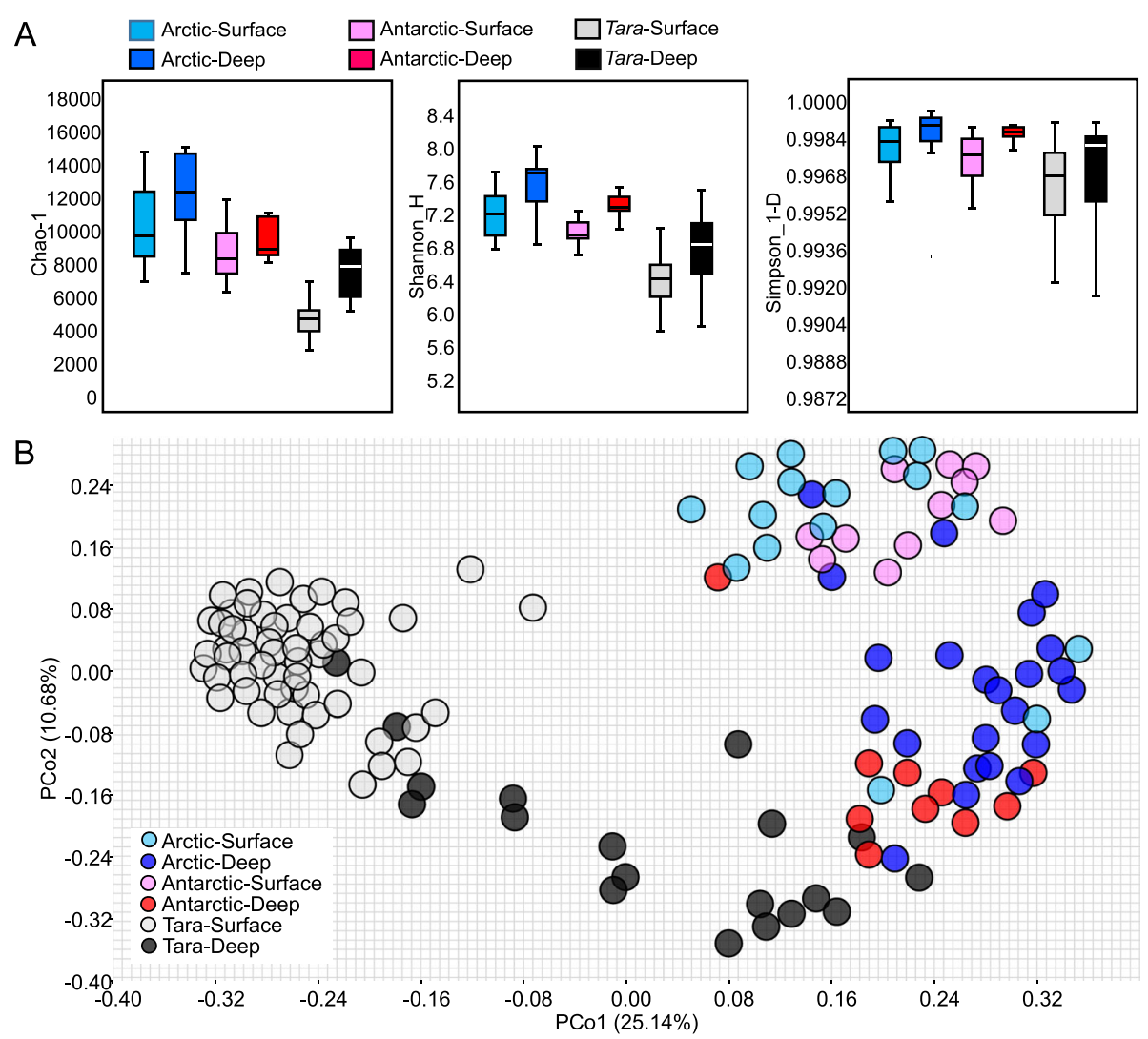

Fig. 3 Alpha- and betadiversity analyses of the polar and Tara Oceans microbiomes. a Chao1, Shannon, and Simpson diversities were calculated based on miTags normalized to the same library size (10,000 sequences per metagenome). $\mathbf{b}$ Jaccard similarity of the microbial communities illustrated by principal coordinates analysis of the OTU matrix (item and tag numbers). This analysis was performed after normalizing the miTags data to the same library size (10,000 sequences per metagenome). The six groups: Arctic-Surface (0-100 m), Arctic-Deep (200-4000 m), AntarcticSurface (0-100 m), Antarctic-Deep (200-4000 m), Tara-Surface (5 m), and Tara-Deep (100-1000 m)

microbial connectivity by major currents [28], whereas the biogeography of deep water communities is largely controlled by ocean circulation [29].

\section{Taxonomic and functional specificity}

The 60 polar metagenomes were combined and the overlapping OTUs between all the polar seawater microbiota and all the Tara seawater microbiota $(n=243)$ were analyzed. The reads were mapped back to the OTUs; of the OTUs with more than 2 reads, 7520 were specific for the polar seawater; of the OTUs with more than 10 reads, 5176 were specific for the polar seawater; and of the OTUs with more than 50 reads, 3221 were specific for the polar seawater; this exceeded the number of OTUs specific for the Tara Oceans seawater (Fig. 4a). The specificity of the polar seawater (i.e., the ratio of polar-specific OTUs to the total number of OTUs present in the polar and non-polar seawater) consistently increased, whereas the specificity of the Tara Oceans seawater decreased with the number of minimum sequences possessed by the OTUs used for examination (Fig. 4b).
When the functions of the polar seawater microbiota were analyzed, a total of 16,638,499 orthologs were derived from the 60 metagenomic assemblies, resulting in a polar marine reference gene catalog (PM-RGC). In comparison with the OM-RGC using BLASTp, 3,903,052 (23.46\%) of the PM-RGC orthologs were specific ( $e$ value $>1 \mathrm{e}-7$ or similarity < 40\%) (Fig. 4c). The orthologs specific for OMRGC or PM-RGC were further searched against the NCBI$\mathrm{Nr}$ database. It was found that more than $97 \%$ of the PMRGC specific orthologs could not be identified ( $e$ value $>1$ e-7 or similarity $<40 \%$ ) in the NCBI-Nr database, while about $61 \%$ of the OM-RGC specific orthologs had no hits ( $e$ value $>1 \mathrm{e}-7$ or similarity $<40 \%$ ) in NCBI-Nr (Fig. $4 \mathrm{~d}$ ).

The OM-RGC and PM-RGC specific orthologs were annotated using the COG database and compared with each other. While the PM-RGC orthologs received 3650 hits in the COG database, the OM-RGC orthologs had 4711 COG hits. Statistical analysis, based on the COG relative abundances (proportion of each COG in the total number of COGs), revealed that 2921 had significantly changed COGs (chi-squared test, $p$ value $<0.05$ ). The 40 most abundant COGs significantly enriched in 

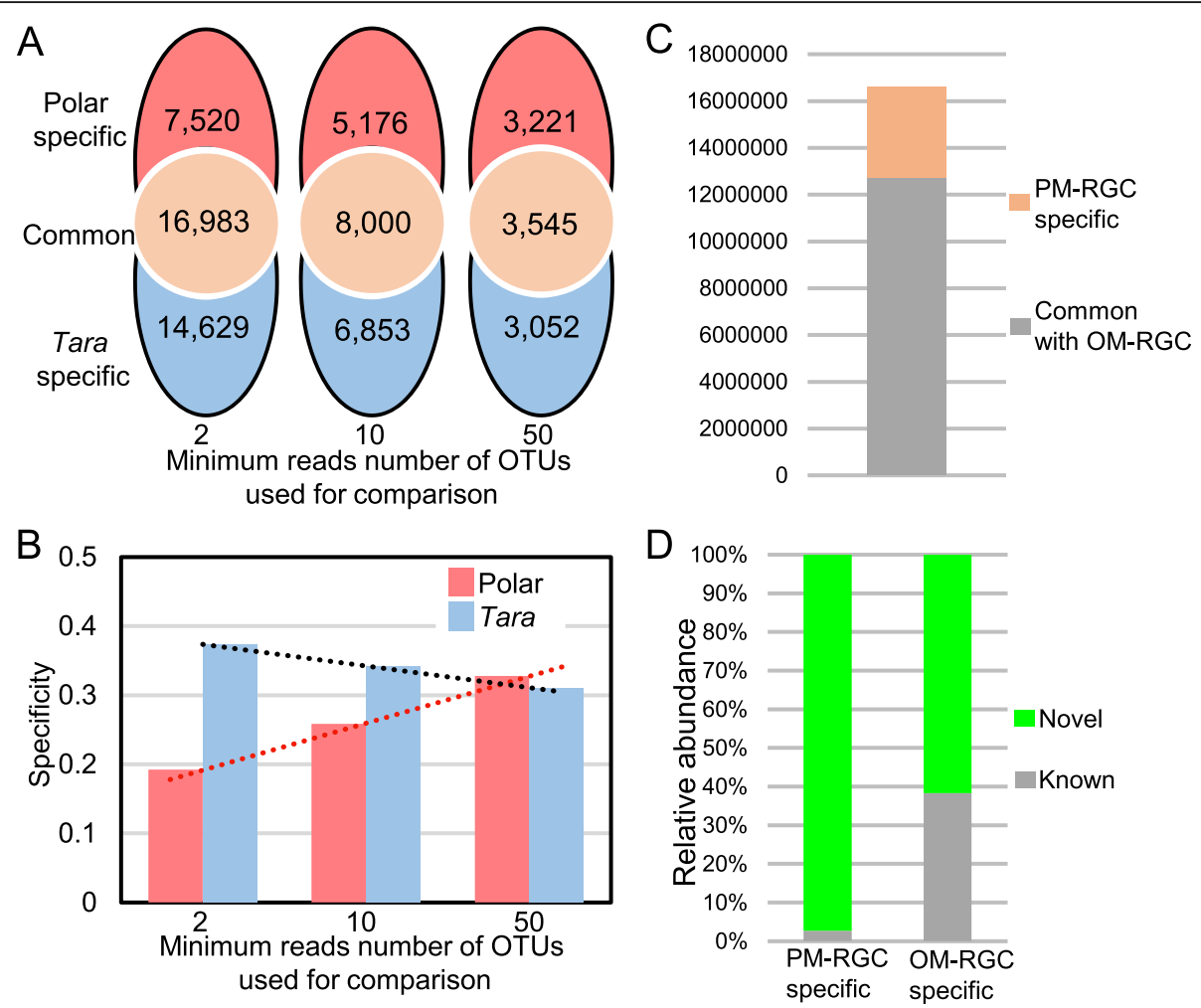

Fig. 4 Comparison between the polar microbiomes and the Tara Oceans data. a Venn diagram showing the distribution of operational taxonomic units (OTUs) across the polar and the Tara miTags. The Tara miTags comprise 16S rRNA sequences extracted from 243 seawater metagenomes. Venn diagrams based on OTUs with more than 2, 10, 50 miTag numbers are shown. $\mathbf{b}$ The change of the taxonomic specificity along with the OTU abundances (indicated by the reads number of OTUs) included for comparison. c Establishment of a polar microbial reference gene catalog (PM-RGC) and comparison with the ocean microbial reference gene catalog (OM-RGC) established by the Tara Oceans study. PM-RGC specific gene orthologs are nonredundant genes that are present in the polar seawater metagenomes but were not detected in the OM-RGC. $\mathbf{d}$ BLASTp searching the NCBI-Nr database using the PM-RGC or OM-RGC specific gene catalog. Genes got hit in NCBI-Nr database are labeled as "known" while genes with no hit are labeled as "novel"

PM-RGC are shown in Fig. 5. These COGs included functions for lipid and sugar metabolism, such as rarelipoprotein B (COG2980), CelD involved in cellulose biosynthesis (COG5653), and sugar phosphate permease (COG2271). Functions for cell membrane biosynthesis, such as the membrane proteins COG4291 and COG4648 without known functions, and membrane proteins involved in dissulfite bond formation (COG5061), were enriched in PM-RGC.

The $16 \mathrm{~S}$ miTags extracted from the Tara Oceans metagenomes mapped to a total of 35,650 OTUs, with the rate of new gene detection at $0.01 \%$ by the end of sampling [1]. However, by sampling both surface and deep polar seawater, a large reservoir of microbial OTUs and functional genes were found that did not overlap with the Tara Oceans datasets. Overall, analyses of the polar seawater metagenomes increased the previously reported microbial diversity by more than $20 \%$. Of the protein-coding genes derived from the polar microbiomes, between 20 and $40 \%$ showed a similarity with the OM-RGC generated by the Tara Oceans datasets. More importantly, 97\% of the PMRGC specific orthologs have unknown functions, and to our knowledge this is the first time to evaluate the overall functional specificity of polar seawater microta based on global seawater datasets. These findings suggest that there is an underestimation of the microbial taxonomic and functional diversity in the global oceans and the existence of specific and function-unknown genes.

\section{Microbes enriched in polar microbiomes and their genomic features}

To further explore novel functional potential of the polar microbiota through genomic analysis, 214 microbial metagenome assembled genomes (MAGs) $(>80 \%$ completeness and $<2 \%$ potential contamination) from the 60 polar metagenomes were recovered. These microbes belonged to 24 different microbial, including Alpha-, Beta-, Gamma-, and Deltaproteobacteria, Bacteroidetes, Actinobacteria, Chloroflexi, Verrucomicrobia, and Parcubacteria (Additional file 1: Figure S2). The distribution of these 214 microbes in polar and non-polar locations was further examined by mapping reads (10 million reads per metagenome) from the 60 polar metagenomes and the 74 Tara Oceans metagenomes; this 


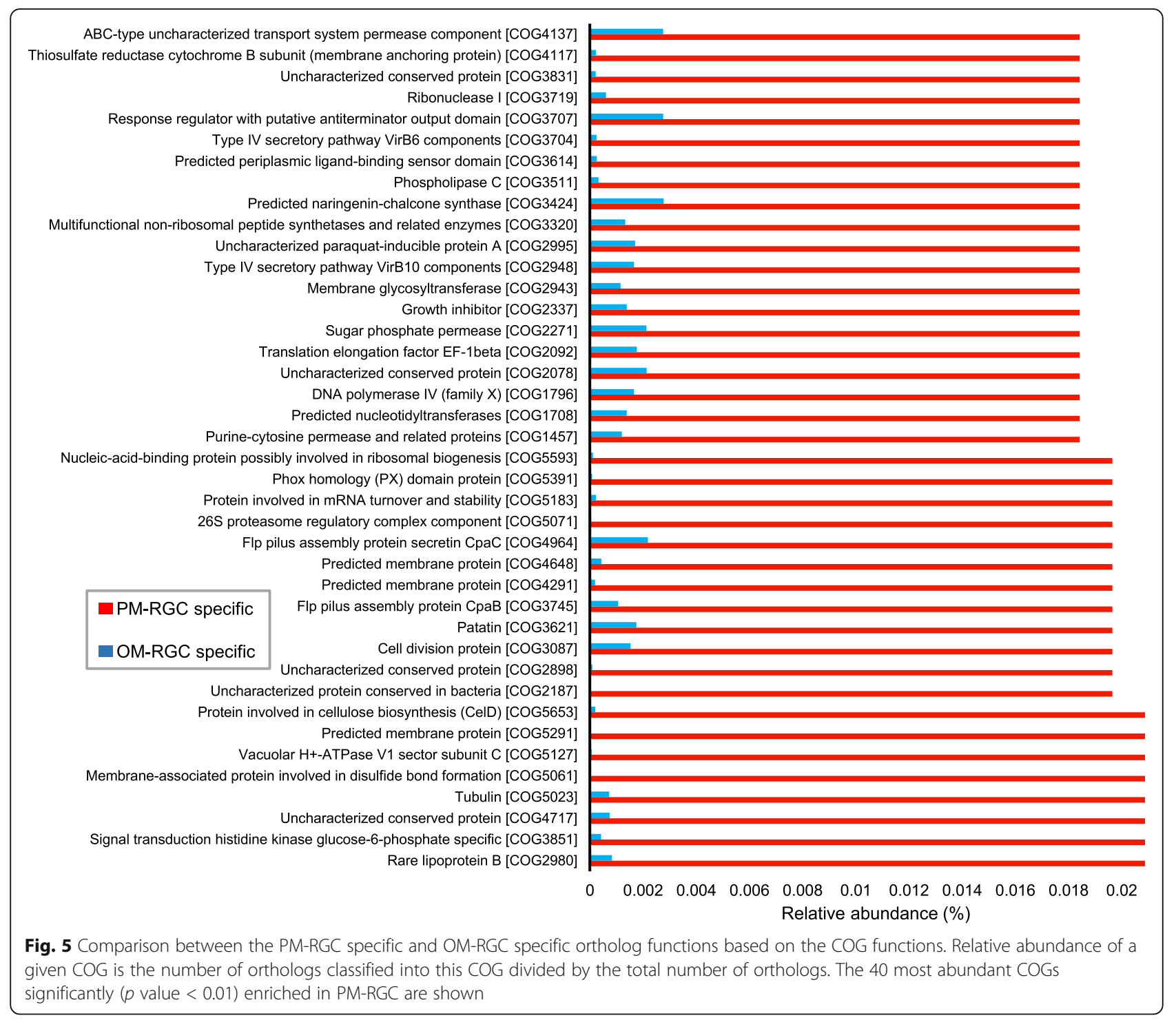

led to the discovery of 32 microbes enriched in polar locations (average coverage in polar metagenomes was $>50$ fold higher than that in Tara metagenomes) (Fig. 6). The microbes enriched in polar metagenomes included members of Alpha- and Gammaproteobacteria, Actinobacteria, Bacteroidetes, Chlamydiae, and Parcubacteria; notably, some taxa (e.g., Parcubacterium sp. Arctic_04) were widespread in most of the polar seawater metagenomes but were almost undetectable in the Tara Oceans metagenomes; moreover, within the Tara Oceans metagenomes, these MAGs tended to be enriched in the deep ocean and almost absent in the surface seawater metagenomes (Fig. 6).

The metabolic pathways of the 32 genomes enriched in polar metagenomes were reconstructed. Based on the KEGG pathway annotation (Fig. 7), several of these genome possessed pathways for nitrogen and sulfur cycling, such as periplasmic nitrate reductase NapA (K02567) and cytochrome c-type protein NapB (K02568) for nitrate reduction, nitrite reductase large subunit nitrite NirB (K00362) and small subunit nitrite NirD (K00363) for nitrite reduction, and sulfate adenylyltransferase subunit 1 CysN (K00956) and subunit 2 CysD (K00957) for sulfate reduction. The C4-dicarboxylic acid pathway was identified as the major pathway adopted by these microbes for carbon fixation. A number of genes for sugar and lipid biosynthesis and uptake were identified in most of the genomes, such as trehalose 6-phosphate phosphatase OtsB (K01087) for trehalose biosynthesis and lipoprotein-releasing system permease proteins (K09808 and K09810) for lipid biosynthesis.

Metagenomic and gneomic analyses in the present study have revealed the functional basis for environmental filtering of microbiota in the polar seawater. Saccharide and lipid biosynthesis genes, enriched in the polar metagenomes 


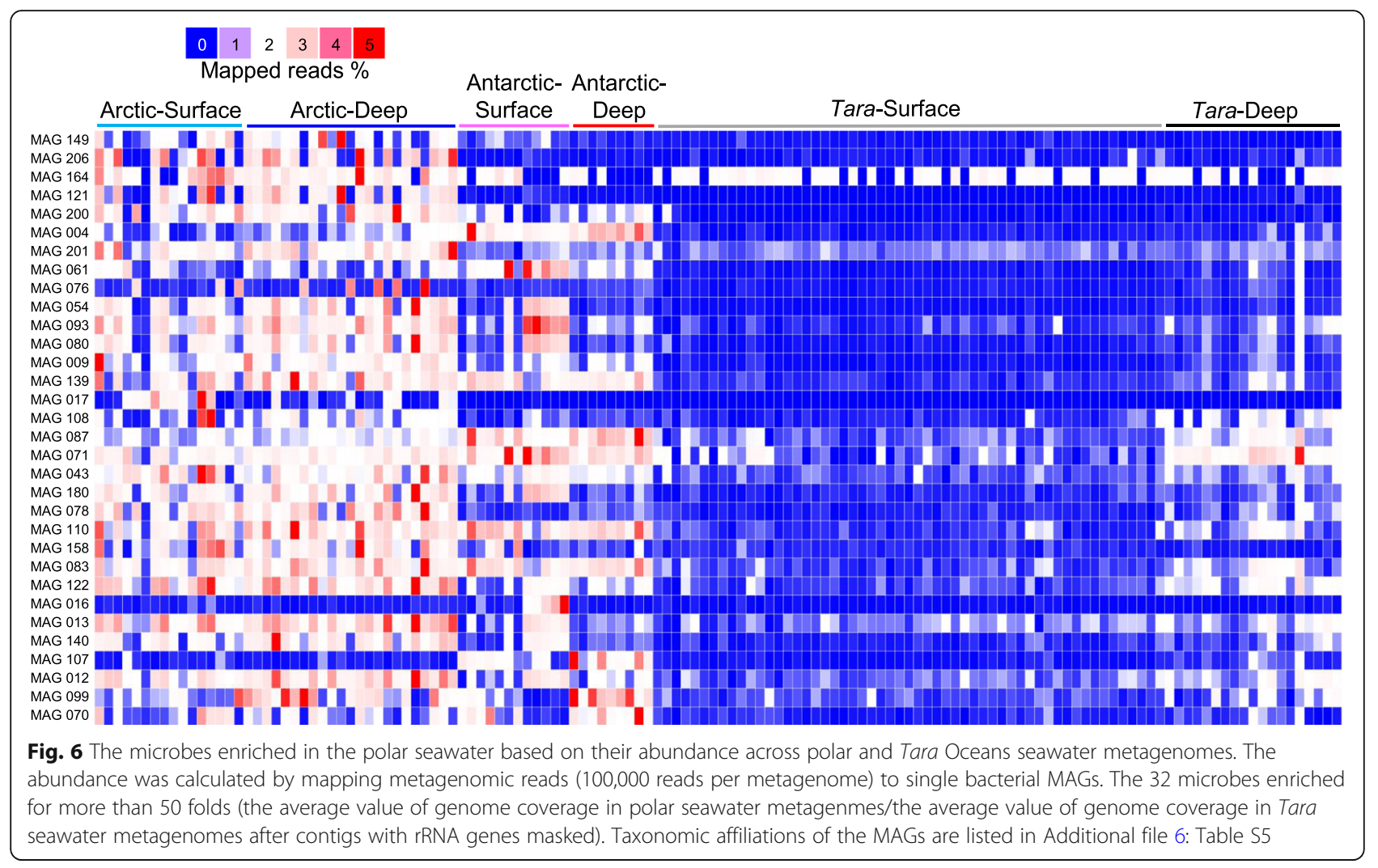

and MAGs, can be used by microbes to combat cold environments. For example, after a temperature decrease from 37 to $16^{\circ} \mathrm{C}$ ("cold shock"), trehalose levels in Escherichia coli cells increased up to 8-fold [30]; biosynthesis of unsaturated fatty acids, which causes a decrease in membrane fluidity of bacterial cells, is crucial to the survival of microbes at low temperature [31-33]. The synthesized polysaccharides can be components of the bacterial cell membrane [34]; consistent with the result that many membrane synthesis related genes are enriched in the polar microbiomes, suggesting their roles in cold adaptation. The C4-dicarboxylic acid pathway also occurs with high frequency in the polar microbes; this pathway does not have as high a demand for coenzymes and metals as other carbon fixation pathways [35] and this may contribute to the success of these microbes in polar regions. In addition, the occurrence of several nitrate and sulfate reduction pathways in certain microbial genomes might be a strategy of metabolic versatility to adapt to environmental change.

\section{Comparison between the Arctic and Antarctic microbiota} In total, 10,754 OTUs were only present in the Arctic microbiota, while 3034 OTUs were only present in the Antarctic microbiota (Additional file 1: Figure S3A). Dereplication of all the ORFs derived from the assembled Arctic metagenomes resulted in 11,806,833 orthologs, and all the assembled Antarctic metagenomes had
$6,460,206$ orthologs. BLASTp searching indicated that 3, $268,783(27.69 \%)$ and $1,160,189(17.96 \%)$ orthologs are unique for the Arctic and Antarctic microbiota, respectively (Additional file 1: Figure S3B and S3C). After annotation, 4170 and 2125 COGs were identified in the Arctic specific and Antarctic specific microbiomes, respectively, and statistical analysis, based on the COG relative abundances, revealed that the two polar regions were enriched with different functions (Additional file 1: Figure S4 and Figure S5). For example, functions related to antibiotic resistance, including vancomycin resistance genes (COG2720), lantibiotic modifying enzyme (COG4403) and exporter of polyketide antibiotics (COG3559) were largely enriched in the Arctic specific orthologs (Additional file 1: Figure S4), whereas functions related to DNA recombination, DNA splicing and RNA transcription were enriched in the Antarctic specific orthologs (Additional file 1: Figure S5).

Despite the notable differences between the polar and temperate microbiomes, implying the existence of microbes that are adapted to the polar environments, comparison between the Arctic and Antarctic microbiomes identified considerable differences in terms of both taxonomy and function. This is consistent with a previous estimation that $78 \%$ of OTUs are unique to the Southern Ocean and 70\% unique to the Arctic Ocean [9]. While more than $50 \%$ of the Arctic OTUs could not be found in the Antarctic microbiomes, only $27.69 \%$ orthologs are specific for the Antarctic 


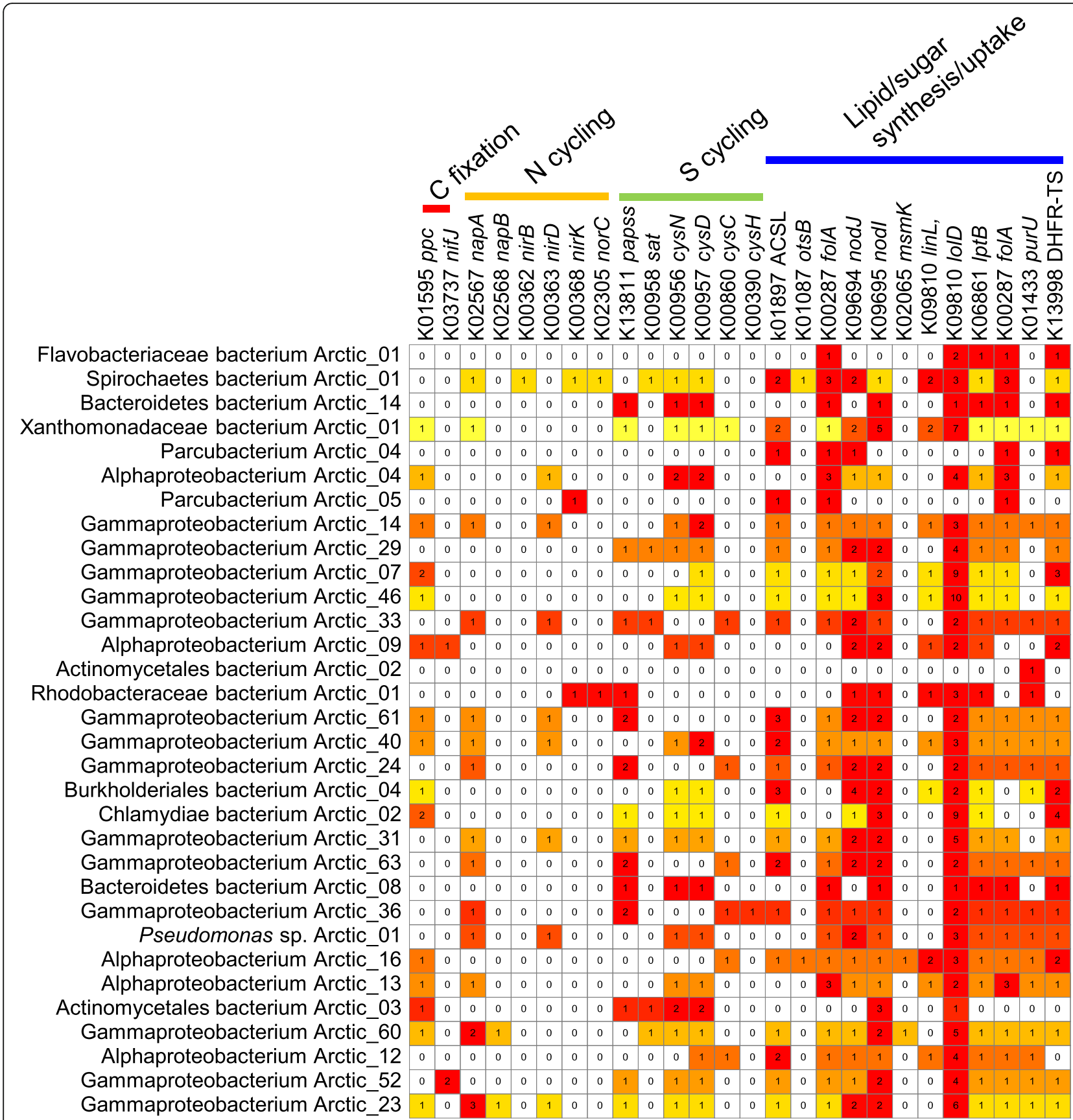

Fig. 7 Carbon fixation, nitrogen cycling, sulfur cycling, and lipid/sugar synthetic genes in the 32 microbes enriched in polar seawater. The genes were annotated by BLASTp searching against the KEGG database

microbiomes. This suggests that the Arctic and Antarctic microbiomes are more similar to each other at the functional level than from a taxonomic perspective. A further comparison suggests that antibiotic biosynthesis, DNA recombination, and DNA splicing are likely to be functions that contribute to the differences between Arctic and Antarctic microbiomes. The greater abundance of antibiotic resistance genes in the Arctic microbiomes might be a result of biological impacts, including human activity, which has already influenced environmental change in the Arctic [36]. DNA recombination and splicing are related to the repair of damaged DNA [37], which can be caused by exposure to ultraviolet radiation; this may be a molecular response to environmental change [38].

\section{Conclusions}

We demonstrate microbial diversity and functional potential found in the polar microbiota and lay a foundation for 
studying the ecology of global ocean microbiota from a more complete perspective. We propose that environmental filtering and microbial dispersal are the major factors shaping the species composition of polar microbiota. Furthermore, we construct here the first functional gene catalog of the polar seawater microbiota, and imply that cold adaptation and environmental change are the major functional basis for microbial community assmebly at the poles.

\section{Materials and methods}

Sampling, DNA extraction, and metagenomic sequencing Seawater samples were collected and filtered through $0.22-\mu \mathrm{m}$ polycarbonate membrane filters (Millipore, MA, USA). The filters were stored in $5 \mathrm{~mL}$ of DNA storage buffer $(500 \mathrm{mM} \mathrm{NaCl}, 50 \mathrm{mM}$ Tris- $\mathrm{HCl}, 40 \mathrm{mM}$ EDTA, and $50 \mathrm{mM}$ glucose) at $-80^{\circ} \mathrm{C}$. The TIANamp Genomic DNA Kit (Tiangen Biotech, Beijing, China) was used to extract DNA from the seawater samples following the manufacturer's protocol.

\section{Metagenomic quality control and assembly}

Quality control of the paired-end reads was performed on a local computer server using the IlluQC.pl script documented in the software NGS QC Toolkit version 2.0 [39]. Reads containing adaptors, poor quality reads (a quality score $<20$ for $>30 \%$ of the read length) or unpaired reads were removed. After quality control, the metagenomic reads $(2,711,430,516$ clean reads with 45,190,509 $\pm 9,900$, 733 clean reads per metagenome) were assembled into contigs (metagenomes were assembled individually) using the software MEGAHIT version 1.0.2 [40] with kmer values with kmer values from 21 to 121 . The OM-RGC datasets, 55 metagenomes of surface seawater and 19 metagenomes of deep seawater in the Tara Oceans project [1] (http:// ocean-microbiome.embl.de/companion.html) were downloaded for comparison. Detailed information about samples and corresponding metagenomic datasets is shown in Additional file 2: Table S1. Information on assembled contigs of polar seawater is shown in Additional file 3: Table S2.

\section{Taxonomic profiling}

$16 \mathrm{~S}$ miTags were recovered from the unassembled metagenomic reads using Parallel-META3 [41]. A HMMER version 3.1 [42] search program documented in ParallelMETA3 was used to predict the $16 \mathrm{~S}$ miTags sequences from both the forward and reverse sequences. The $16 \mathrm{~S}$ miTags were mapped to a database that integrates GreenGenes [43] with RDP [44] and SILVA [45] using the software Bowtie2 [46] and contains OTUs picked at 97\% similarity or above. To reduce the false discovery rate due to sequencing errors, in a metagenome, only OTUs with two or above read numbers were returned (thus all singletons were removed). Phylum (class for Proteobacteria) and genus profiles were generated for the 60 polar seawater metagenomes using the OTU-taxa map documented in Parallel-META3 (PM-pipeline). The full list of phyla (class for Proteobacteria) and genera based on $16 \mathrm{~S}$ miTags are given in Additional file 4: Table S3 and Additional file 5: Table S4, respectively.

\section{Venn analysis}

The overlapped OTUs between the polar microbiomes and Tara Oceans microbiomes (Tara Oceans miTags includes 16S rRNA gene sequences extracted from 243 metagenomes) were calculated using the online Venn program (http://bioinformatics.psb.ugent.be/webtools/Venn/ ), where the lists of OTU identities were uploaded as input. The polar and Tara Oceans seawater specificity was calculated based on the formulas:

$$
\begin{aligned}
& \text { Polar specificity }=\frac{\text { Number of polar-specific OTUs }}{\text { Number of OTUs in polar and Tara samples }} \\
& \text { Tara specificity }=\frac{\text { Number of Tara-specific OTUs }}{\text { Number of OTUs in polar and Tara samples }}
\end{aligned}
$$

\section{Alpha- and beta-diversity analyses}

Alpha- and beta-diversity analyses were performed on the polar and Tara Oceans seawater metagenomes using normalized data sets. 10,000 $16 \mathrm{~S}$ miTags were extracted from each metagenome using the software Seqtk (https://github.com/lh3/seqtk). The following OTUs classification was performed as described above. Chao1, Shannon, and Simpson diversity were calculated using the alpha_diversity.py script implemented in QIIME [47]. Jaccard distances, which are based on the presence/ absence of OTUs, were generated using the OTU table for analyses of the community similarities, and visualized by PCoA (transformation exponent $=2$ ) implemented in the software PAST version 2.0 [48].

\section{Functional analyses of the polar seawater microbiota} ORFs were predicted from the assembled contigs using MetaGeneMark version 2.8 (genes on both strands were predicted; gene overlaps were allowed; cutoff in probability of initiation and termination in non-coding state was 0.5 ). Protein sequences derived from the 60 polar seawater metagenomes were combined and subjected to CD-HIT (> 95\% sequence identity) to generate a nonredundant catalog called PM-RGC. The PM-RGC orthologs sequences (minimum length 100) were BLASTp ( $e$ value $<1 \mathrm{e}-7$; $>60 \%$ sequence identity for $>60 \%$ of the length of the query sequences; maximum hit number $=1$ ) searched against the OM-RGC [1] for PM-RGC specific orthologs. To evaluate the percentage of known proteins, the PM-RGC orthologs were DIAMOND [49] BLASTp ( $e$ value $<1 \mathrm{e}-7$; $>60 \%$ sequence 
identity for $>60 \%$ of the length of the query sequences; maximum hit number $=1$ ) searched against the NCBI-Nr database. For functional annotation and classification, the PM-RGC specific and OM-RGC specific orthologs were BLASTp $(e$ value $<1 \mathrm{e}-7$; $>60 \%$ sequence identity for $>60 \%$ of the length of the query sequences; maximum hit number $=1$ ) searched against the COG database [50]. Statitical analyses were performed using chi-squared test $(p$ value $<0.05$ as significance cutoff).

\section{Genome binning and analyses}

Genome binning was performed with contigs using the software MaxBin2 [51] and MetaBAT [52]. To generate coverage profiles for the assembled contigs, reads from a single metagenome were mapped to the corresponding assembly using Bowtie2 (input reads were in fastq format; the script bowtie2-build was used). The output files were visualized in SAMtools version 1.10 [53] (input files were in SAM format; the scripts view, sort, and depth were used). The coverage profiles and assembled contigs were subject to the binning software MaxBin2 [51] (minimum probability for algorithm 0.8; 107 marker genes) to separate the contigs. The resulting contig groups were subjected to a second binning process using MetaBAT [52] for further separation to get MAGs. The output MAGs were checked for completeness and contamination using CheckM (lineage_wf) [54]. All the MAGs were compared for the average nucleotide identity using pyani [55] (ANIb model), and MAGs with an ANI value higher than 0.99 were considered duplicates. Taxanomy designation of the MAGs was performed using using the software GTDB-Tk v0.3.2 [56] (the script classify_wf was used). Information of the MAGs is presented in Additional file 6: Table S5.

For genome annotation, ORFs were predicted using Prodigal (single genome model; close end) and then BLASTp searched against the KEGG database $(e$ value $<1 \mathrm{e}-7$; $>60 \%$ sequence identity for $>60 \%$ of the length of the query sequences). Metabolic pathways in a single bacterium were reconstructed using the online tool KEGG Mapper (https:// www.genome.jp/kegg/mapper.html). Genes under the categories carbohydrate metabolism and energy production were analyzed. The rRNA genes in the MAGs were predicted using the software Barrnap (https://github.com/tseemann/barrnap), under both bacteria and archaea models. The coverage profiles of the MAGs in metagenomes were calculated by Bowtie 2 and SAMtools, after masking the contigs with rRNA genes.

\section{Data availability}

All metagenomic datasets and gene catalog of PM-RGC have been deposited in the NCBI database (BioProject accession no. PRJNA588686). The 214 MAGs have been uploaded to figshare (https://figshare.com/s/fd5f60b5da7a63aaa74b) and Genbank of NCBI (BioProject accession no. SUB7116349).
The PM-RGC catalog can also be downloaded at https://figshare.com/s/28fbf48ffadfade8a77f, https://figshare.com/s/2f8 d1ce9fb2d68f76bb2, and https://figshare.com/s/28fbf48ffadf ade8a77f.

\section{Supplementary information}

Supplementary information accompanies this paper at https://doi.org/10. 1186/s40168-020-00826-9.

Additional file 1: Figure S1. Genus-level composition based on analysis of $16 \mathrm{~S}$ miTags. Abundant genera (the top 30 genera in terms of maximum relative abundance) are shown with all other genera grouped together as 'Minor or unclassified'. The four groups: Arctic-Surface (0-100 m), Arctic-Deep (200-4000 m), Antarctic-Surface (0-100 m), and Antarctic-Deep (200-4000 m). Figure S2. Taxonomic distribution of the 214 MAGs recovered from the polar metagenomes. All the MAGs have $80 \%$ or higher completeness. Figure S3. Comparison between the Arctic and Antarctic microbiomes. A Venn diagram showing the distribution of OTUs across the Arctic and the Antarctic miTags. Only OTUs with more than 2 miTag numbers are included for analysis. B BLASTp searching Antarctic orthologs using the Arctic orthologs as queries. C BLASTp searching Arctic orthologs using the Antarctic orthologs as queries. Orthologs of lower than $40 \%$ similarity or higher than 1e-7 e-value were considered as 'specific'. Figure S4. Functions enriched in the Arctic microbiomes. The Arctic and Antarctic-specific orthologs were annotated by searching against the COG database. Relative abundance of a given COG is the number of orthologs classified into this COG divided by the total number of orthologs. The 40 most abundant COGs significantly ( $p$-value <0.01) enriched in the Arctic are shown. Orange color indicates antibiotic resistance genes. Figure S5. Functions enriched in the Antarctic microbiomes. The Arctic and Antarctic-specific orthologs were annotated by searching against the COG database. Relative abundance of a given COG is the number of orthologs classified into this COG divided by the total number of orthologs. The 40 most abundant COGs significantly ( $p$-value $<0.01$ ) enriched in the Antarctic are shown. Purple color indicates genes involved in DNA recombination.

Additional file 2: Table S1. Sampling locations and metagenomes information.

Additional file 3: Table S2. Information of the assembled contigs. Additional file 4: Table S3. Full phyla (class for Proteobacteria) list and their abundance in the polar seawater.

Additional file 5: Table S4. Full list of genera and their abundance in the polar seawater.

Additional file 6: Table S5. Information of the MAGs extracted from polar seawater metagenomes.

\section{Abbreviations}

OM-RGC: Ocean microbial reference gene catalog; OTUs: Operational taxonomic units; PCoA: Principal coordinates analysis; PM-RGC: Polar marine reference gene catalog

\section{Acknowledgements}

Not applicable.

\section{Authors' contributions}

$Y Z Z, J H, X L C, Q L Q, B B X$, and MW conceived the study. WZ, WD, MW, SF, and BY performed data analyses. SC collected samples. WZ and AM wrote the manuscript. The authors read and approved the final manuscript.

\section{Funding}

This work was funded by the National Key R \& D Program of China (2018YFC1406700), the Scientific Research Staring Foundations of Ocean University of China (841912034 and 841912035), The response and feedback of the Southern Ocean to climate change (RFSOCC2020-2025), the National Science Foundation of China (41976230, 41906201, 41476168, U1706207, 31630012, 91851205, 31670063), the Major Scientific and Technological Innovation Project of Shandong Province (2019JZZY010817), the Program of 
Shandong for Taishan Scholars (tspd20181203), and AoShan Talents Cultivation Program Supported by Qingdao National Laboratory for Marine Science and Technology (2017ASTCP-OS14).

\section{Ethics approval and consent to participate} Not applicable.

\section{Consent for publication}

Not applicable.

\section{Competing interests}

We have no conflicts of interest to disclose.

\section{Author details}

${ }^{1}$ The Key Laboratory for Polar Science MNR, Polar Research Institute of China, Shanghai 200136, China. ${ }^{2}$ College of Marine Life Science, Frontiers Science Center for Deep Ocean Multispheres and Earth System, Ocean University of China, Qingdao 266003, China. ${ }^{3}$ State Key Laboratory of Microbial Technology, and Marine Biotechnology Research Center, Shandong University, Qingdao 266237, China.

\section{Received: 17 December 2019 Accepted: 13 March 2020} Published online: 02 April 2020

\section{References}

1. Sunagawa $\mathrm{S}$, et al. Structure and function of the global ocean microbiome. Science. 2015;348:1261359.

2. Louca S, Parfrey LW, Doebeli M. Decoupling function and taxonomy in the global ocean microbiome. Science. 2016:353:1272-7.

3. Haro-Moreno JM, López-Pérez M, José R, Picazo A, Camacho A, RodriguezValera F. Fine metagenomic profile of the Mediterranean stratified and mixed water columns revealed by assembly and recruitment. Microbiome. 2018;6:128.

4. Dombrowski N, Seitz KW, Teske AP, Baker BJ. Genomic insights into potential interdependencies in microbial hydrocarbon and nutrient cycling in hydrothermal sediments. Microbiome. 2017;5:106.

5. Liu J, et al. Proliferation of hydrocarbon-degrading microbes at the bottom of the Mariana Trench. Microbiome. 2019;7:1-13.

6. Jones HJ, Kröber E, Stephenson J, Mausz MA, Jameson E, Millard A, Purdy KJ Chen Y. A new family of uncultivated bacteria involved in methanogenesis from the ubiquitous osmolyte glycine betaine in coastal saltmarsh sediments. Microbiome. 2019:7:1-11.

7. Ding W, Zhang W, Wang R, Sun Y, Pei B, Gao Z, Qian PY. Distribution, diversity and functional dissociation of the mac genes in marine biofilms. Biofouling. 2019;35:230-43.

8. $\quad$ Ding W, Zhang W, Alikunhi NM, Batang Z, Pei B, Wang R, Chen L, AlSuwailem A, Qian PY. Metagenomic analysis of zinc surface-associated marine biofilms. Micro Ecol. 2019;77:406-16.

9. Zhang $W$, et al. Marine biofilms constitute a bank of hidden microbial diversity and functional potential. Nat Commun. 2019;10:1-10.

10. Pita L, Rix L, Slaby BM, Franke A, Hentschel U. The sponge holobiont in a changing ocean: from microbes to ecosystems. Microbiome. 2018:6:46.

11. Zhang $W$, et al. Gut microbial divergence between two populations of the hadal amphipod Hirondellea gigas. Appl Environ Microbiol. 2019;85 e02032-18.

12. Osman EO, Suggett DJ, Voolstra CR, Tye Pettay D, Clark DR, Pogoreutz C, Sampayo EM, Warner ME, Smith DJ. Coral microbiome composition along the northern Red Sea suggests high plasticity of bacterial and specificity of endosymbiotic dinoflagellate communities. Microbiome. 2020;8:1-16.

13. Hodson A, Anesio AM, Tranter M, Fountain A, Osborn M, Priscu J, LaybournParry J, Sattler B. Glacial ecosystems. Ecol Monogr. 2008;78:41-67.

14. Verde C, Giordano D, Bellas CM, di Prisco G, Anesio AM. Polar marine microorganisms and climate change. Adv Microb Physiol. 2016;69:187-215.

15. Morison J, Kwok R, Peralta-Ferriz C, Alkire M, Rigor I, Andersen R, Steele M. Changing arctic ocean freshwater pathways. Nature. 2012;481:66.

16. Purkey SG, Johnson GC. Antarctic bottom water warming and freshening: contributions to sea level rise, ocean freshwater budgets, and global heat gain. J Clim. 2013;26:6105-22.

17. Najafi MR, Zwiers FW, Gillett NP. Attribution of Arctic temperature change to greenhouse-gas and aerosol influences. Nat Clim Chang. 2015;5:246-9.
18. Elder CD, Xu X, Walker J, Schnell JL, Hinkel KM, Townsend-Small A, Arp CD, Pohlman JW, Gaglioti BV, Czimczik Cl. Greenhouse gas emissions from diverse Arctic Alaskan lakes are dominated by young carbon. Nat Clim Chang. 2018;8:166-71.

19. Turner J, Overland JE, Walsh JE. An Arctic and Antarctic perspective on recent climate change. Int J Climatol. 2007;27:277-93.

20. Walsh JE. A comparison of Arctic and Antarctic climate change, present and future. Antarct Sci. 2009;21:179-88.

21. Maksym T. Arctic and Antarctic Sea ice change: contrasts, commonalities, and causes. Ann Rev Mar Sci. 2019;11:187-213.

22. Parkinson $\mathrm{CL}$, DiGirolamo NE. New visualizations highlight new information on the contrasting Arctic and Antarctic sea-ice trends since the late 1970s. Remote Sens Enviro. 2016;183:198-204.

23. Galand PE, Casamayor EO, Kirchman DL, Lovejoy C. Ecology of the rare microbial biosphere of the Arctic Ocean. Proc Natl Acad Sci USA. 2009;106:22427-32.

24. Ghiglione JF, et al. Pole-to-pole biogeography of surface and deep marine bacterial communities. Proc Natl Acad Sci USA. 2012;109:17633-8.

25. Kleinteich J, Hildebrand F, Bahram M, Voigt AY, Wood SA, Jungblut AD, Küpper FC, Quesada A, Camacho A, Pearce DA, et al. Pole-to-pole connections: similarities between Arctic and Antarctic microbiomes and their vulnerability to environmental change. Front Ecol Evol. 2017;5:137.

26. Sul WJ, Ta O, Ducklow HW, La A-Z, Sogin ML. Marine bacteria exhibit a bipolar distribution. Proc Natl Acad Sci USA. 2013;110:2342-7.

27. Pommier T, Douzery EJ, Mouillot D. Environment drives high phylogenetic turnover among oceanic bacterial communities. Biol Lett. 2012;8:562-6.

28. Cavicchioli R. Microbial ecology of Antarctic aquatic systems. Nat Rev Microbiol. 2015;13:691.

29. Salazar G, Cornejo-Castillo FM, Benítez-Barrios V, Fraile-Nuez E, Álvarez-Salgado XA, Duarte CM, Gasol JM, Acinas SG. Global diversity and biogeography of deep-sea pelagic prokaryotes. ISME J. 2016;10: 596-608.

30. Kandror O, DeLeon A, Goldberg AL. Trehalose synthesis is induced upon exposure of Escherichia coli to cold and is essential for viability at low temperatures. Proc Natl Acad Sci USA. 2002;99:9727-32.

31. Nichols DS, Miller MR, Davies NW, Goodchild A, Raftery M, Cavicchioli R. Cold adaptation in the Antarctic archaeon Methanococcoides burtonii involves membrane lipid unsaturation. J Bacteriol. 2004;186:8508-15.

32. Shivaji S, Prakash JS. How do bacteria sense and respond to low temperature? Arch Microbiol. 2010;192:85-95.

33. Sohlenkamp C, Geiger O. Bacterial membrane lipids: diversity in structures and pathways. FEMS Microbiol Rev. 2016;40:133-59.

34. Turner RD, Mesnage S, Hobbs JK, Foster SJ. Molecular imaging of glycan chains couples cell-wall polysaccharide architecture to bacterial cell morphology. Nat Commun. 2018;9:1263.

35. Berg IA. Ecological aspects of the distribution of different autotrophic $\mathrm{CO}_{2}$ fixation pathways. Appl Environ Microbiol. 2011;77:1925-36.

36. Meie WN, et al. Arctic sea ice in transformation: a review of recent observed changes and impacts on biology and human activity. Rev Geophys. 2014;52: 185-217.

37. Muñoz MJ, Pérez Santangelo MS, Paronetto MP, de la Mata M, Pelisch F, Boireau S, Glover-Cutter K, Ben-Dov C, Blaustein M, Lozano JJ. DNA damage regulates alternative splicing through inhibition of RNA polymerase ॥ elongation. Cell. 2009;137:708-20.

38. MacFadyen EJ, Williamson CE, Grad G, Lowery M, Jeffrey WH, Mitchell DL. Molecular response to climate change: temperature dependence of UVinduced DNA damage and repair in the freshwater crustacean Daphnia pulicaria. Glob Chang Biol. 2004;10:408-16.

39. Patel Ravi K, Mukesh J. NGS QC Toolkit: a toolkit for quality control of next generation sequencing data. PloS One. 2012;7:e30619.

40. Li D, Liu CM, Luo R, Sadakane K, Lam TW. MEGAHIT: an ultra-fast singlenode solution for large and complex metagenomics assembly via succinct de Bruijn graph. Bioinformatics. 2015;31:1674-6.

41. Jing G, Sun Z, Wang H, Gong Y, Huang S, Ning K, Xu J, Su X. Parallel-META 3: comprehensive taxonomical and functional analysis platform for efficient comparison of microbial communities. Sci Rep. 2017;7:40371.

42. Eddy SR. Accelerated profile HMM searches. PLoS Comput Biol. 2011;7: e1002195.

43. DeSantis TZ, Hugenholtz P, Larsen N, Rojas M, Brodie EL, Keller K, Huber T, Dalevi D, Hu P, Andersen GL. Greengenes, a chimera-checked 165 rRNA gene database and workbench compatible with ARB. Appl Environ Microbiol. 2006;72:5069-72. 
44. Cole JR, Wang Q, Fish JA, Chai B, McGarrell DM, Sun Y, Brown CT, PorrasAlfaro A, Kuske CR, Tiedje JM. Ribosomal Database Project: data and tools for high throughput rRNA analysis. Nucleic Acids Res. 2013;42:633-42.

45. Quast C, et al. The SILVA ribosomal RNA gene database project: improved data processing and web-based tools. Nucleic Acids Res. 2012;41:590-6.

46. Langmead B, Salzberg SL. Fast gapped-read alignment with Bowtie 2. Nat Methods. 2012;9:357-9

47. Caporaso JG, et al. QIIIME allows analysis of high-throughput community sequencing data. Nat Methods. 2010;7:335.

48. Hammer $\varnothing$, Harper DAT, Ryan PD. PAST: paleontological statistics software package for education and data analysis. Palaeontol Electron. 2001:4:1-9.

49. Buchfink B, Xie C, Huson DH. Fast and sensitive protein alignment using DIAMOND. Nat Methods. 2015;12:59.

50. Galperin MY, Makarova KS, Wolf Yl, Koonin EV. Expanded microbial genome coverage and improved protein family annotation in the COG database. Nucleic Acids Res. 2014;43:261-9.

51. Wu YW, Simmons B, Singer SW. MaxBin 2.0: an automated binning algorithm to recover genomes from multiple metagenomic datasets. Bioinformatics. 2016;32:605-7.

52. Kang DD, Froula J, Egan R, Wang Z. MetaBAT, an efficient tool for accurately reconstructing single genomes from complex microbial communities. Peer J. 2015:3:e1165.

53. Li $\mathrm{H}$, et al. The sequence alignment/map format and SAMtools. Bioinformatics. 2009;25:2078-9.

54. Parks DH, Imelfort M, Skennerton CT, Hugenholtz P, Tyson GW. CheckM: assessing the quality of microbial genomes recovered from isolates, single cells, and metagenomes. Genome Res. 2015;25:1043-55.

55. Pritchard L, Glover RH, Humphris S, Elphinstone JG, Toth IK. Genomics and taxonomy in diagnostics for food security: soft-rotting enterobacterial plant pathogens. Anal Methods. 2016;8:12-24.

56. Chaumeil PA, Mussig AJ, Hugenholtz P, Parks DH. GTDB-Tk: a toolkit to classify genomes with the Genome Taxonomy Database. Bioinformatics. 2020:36:1925-27.

\section{Publisher's Note}

Springer Nature remains neutral with regard to jurisdictional claims in published maps and institutional affiliations.

Ready to submit your research? Choose BMC and benefit from:

- fast, convenient online submission

- thorough peer review by experienced researchers in your field

- rapid publication on acceptance

- support for research data, including large and complex data types

- gold Open Access which fosters wider collaboration and increased citations

- maximum visibility for your research: over $100 \mathrm{M}$ website views per year

At $\mathrm{BMC}$, research is always in progress.

Learn more biomedcentral.com/submissions 